\title{
Microbolometer matrix gain non-uniformity determination and correction with the aid of ring and spot infrared emitters
}

by R. Olbrycht* and B. Więcek*

*Institute of Electronics, Technical University of Lodz, 90-924 Lodz, Poland, wiecek@p.lodz.pl

\section{Abstract}

The Authors propose a method for determination of microbolometer matrix gain non-uniformity with the aid of infrared emitters built into an infrared camera. This paper presents the research results on this subject with two types of infrared emitters - ring and spotlight. Obtained results are in favour of the ring emitter - in case of spotlight there is a risk of unwanted artifacts. Periodical determination of microbolometers' gain values in thermal camera enables verification that these remain unchanged. In case of detection that gain values changed, it is possible to compensate for observed differences.

\section{Introduction}

Gain values of all microbolometers in a matrix should remain unchanged in a given measurement range and under stable Peltier temperature. Therefore in many thermal imagers gain non-uniformity correction is done at the camera production stage, and the camera itself cannot update this correction. Only thermal drift is periodically corrected during the camera operation with help of the opaque optical shutter, and this is an automated process independent on the user. The proposed method of gain non-uniformity determination relies upon using an additional source of infrared radiation (the emitter) built into the thermal camera. The emitter is driven by electrical current, and during the normal camera operation it remains unpowered. Every desired period of time (e.g. 1 month) it may be turned on to verify if gain values (in other words - gain non-uniformity) of all microbolometers in a matrix remain stable over this period. In case of detection of changes, it is possible to compensate for observed differences.

\section{Infrared emitters}

Authors researched two types of infrared emitters: ring and spotlight. The ring emitter was made from thin metal, it is shown in figure 1(a). It should be highly emissive towards the microbolometer matrix. To achieve this effect, its surface was covered with black matte paint $(\varepsilon \approx 0.93 \pm 0.03)$. From the other side of the emitter it is preferred to reduce the amount of radiated energy by leaving the metal surface without any covering layer $(\varepsilon \approx 0,09 \pm 0,05)$. This difference between both sides (having the same temperature value) is revealed by thermograms shown in figure 1(b,c). The inner and outer radius of the ring emitter, as well as its distance from the matrix were found with the aid of simulations described in chapter 4.

The spotlight emitter in TO-5 package - shown in figure 2(a) - is MIRL 17-900 element made by Intex using MEMS technology. Its active area $\left(1,7 \times 1,7 \mathrm{~mm}^{2}\right)$ is made of amorphous carbon, and has emissivity value $\varepsilon \approx 0.8$. Under maximum recommended voltage of $7 \mathrm{~V}$ it rapidly heats up to $750^{\circ} \mathrm{C}$. More technical specifications can be found in its technical documentation [4].

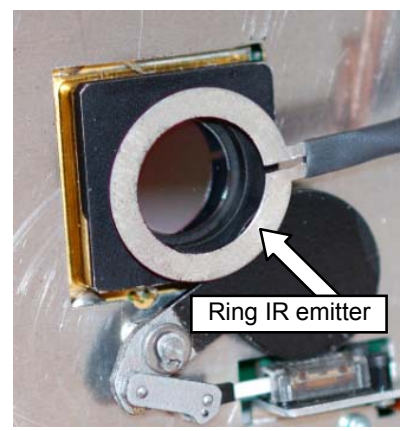

(a)

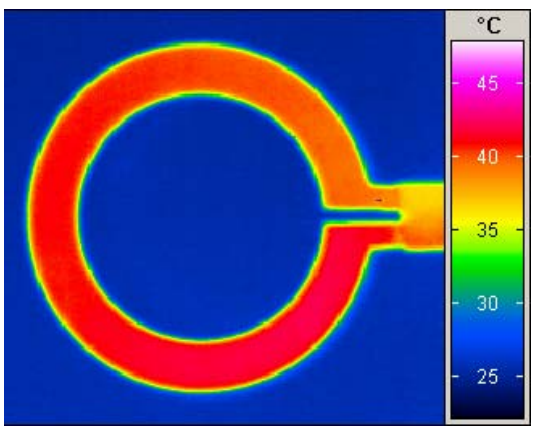

(b)

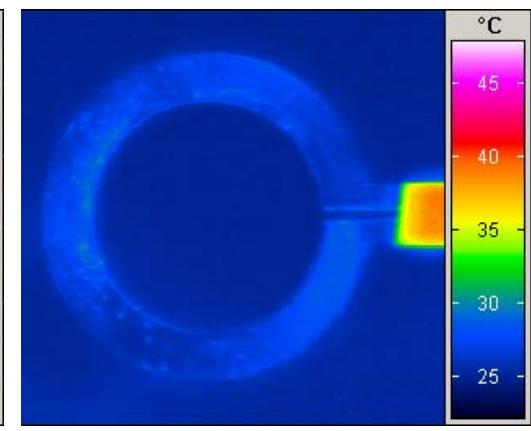

(c)

Fig. 1. (a) Placement of ring infrared emitter inside the camera; thermogram of (b) emissive, (c) reflective surface of the emitter 


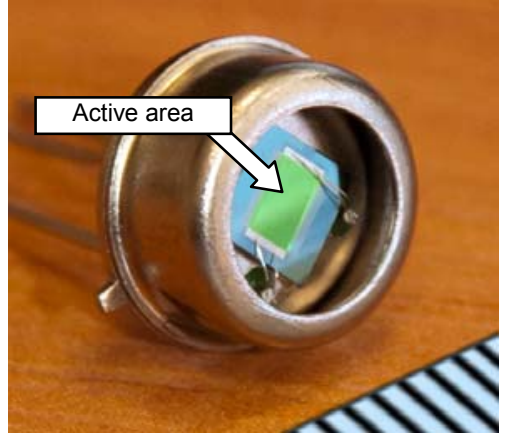

(a)

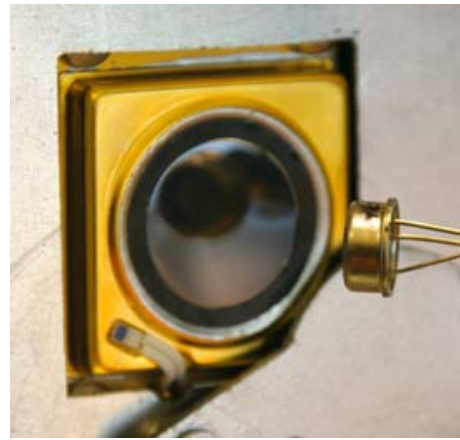

(b)

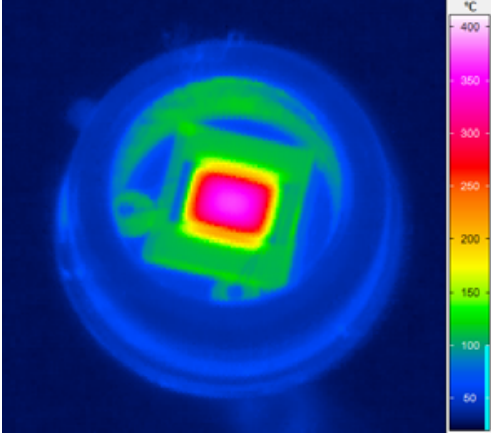

(c)

Fig. 2. (a) Spotlight infrared emitter; (b) emitter placement inside camera; (c) emitter thermogram

\section{Determination of the gain non-uniformity}

To determine the gain non-uniformity with the proposed method it is required to acquire two series of thermograms - with emitter turned off (equation 1) and on (equation 2). Later on both series should be averaged in the time domain (equation 3) to obtain two averaged thermograms. This is to reduce instantaneous noise. The requirement is that during the acquisitions the camera should be pointed to a stationary scene [1]. The difference between the two averaged thermograms (equation 4) contains the information about the gain non-uniformity, but it is altered by the uneven illumination of matrix by the emitter. To reduce this problem, the authors simulated the radiative heat transfer between emitters and the matrix, and found out the optimal distances: matrix to ring emitter and matrix to spot emitter. Keeping these distances ensures the lowest differences in illumination pattern. Next, keeping determined distances, illumination patterns were measured for each emitter. Knowing these patterns it was possible to compensate for uneven illumination (equation 5), and determine real values of microbolometers' gain values. As a reference, traditional two-point non-uniformity correction was done with two blackbodies, and the authors analyzed the differences between the obtained (figure $3 b, c$ ) and reference gain values (figure $3 a$ ) for all microbolometers.

$$
\begin{aligned}
& Y_{i j}^{t_{1}}\left(M_{e, \lambda}^{\text {scene }}\right)=G_{i j} M_{e, \lambda}^{\text {scene }}+O_{i j} \\
& Y_{i j}^{t_{1}}\left(M_{e, \lambda}^{\text {scene }}+M_{e, \lambda}^{\text {emitter }}\right)=G_{i j}\left(M_{e, \lambda}^{\text {scene }}+M_{e, \lambda}^{\text {emitter }}\right)+O_{i j} \\
& Y_{i j}^{a v g\left(t_{1} \ldots t_{n}\right)}=\frac{\sum_{a=1}^{n} Y_{i j}^{t_{a}}}{n} \\
& G_{i j}=\frac{Y_{i j}^{a v g\left(t_{1} \ldots t_{n}\right)}\left(M_{e, \lambda}^{\text {scene }}+M_{e, \lambda}^{\text {emitter }}\right)-Y_{i j}^{a v g\left(t_{1} \ldots t_{n}\right)}\left(M_{e, \lambda}^{\text {scene }}\right)}{M_{e, \lambda}^{\text {emitter }}} \\
& G_{i j}=\frac{w_{i j}\left[\overline{Y\left(t_{2}\right)}-\overline{Y\left(t_{1}\right)}\right]}{\Delta Y} \\
& \Delta Y=\frac{\sum_{i=1}^{M} \sum_{j=1}^{N}\left[Y_{i j}^{a v g\left(t_{1} \ldots t_{n}\right)}\left(M_{e, \lambda}^{\text {scene }}+M_{e, \lambda}^{e m i t t e r}\right)-Y_{i j}^{a v g\left(t_{1} \ldots t_{n}\right)}\left(M_{e, \lambda}^{\text {scene }}\right)\right]}{M N}
\end{aligned}
$$

where $M$ stands for the number of columns in the matrix, and $N$ - rows. 


\section{Simulation of matrix illumination energy and unevenness}

To simulate the heat transfer between the surfaces of emitter and matrix, the authors divided those surfaces into differential elements $\mathrm{dA}_{1}$ and $\mathrm{dA}_{2}$, as shown in Figure 3. Each element was assumed to have uniform temperature distribution because of very small area. Hence it was possible to create the model of radiative meat transfer between the emitter and the matrix using configuration factors calculated individually for every pair $\left(d_{A_{1}}, d A_{2}\right)$ using equation (7). Calculated values are proportional to the intensity of radiative heat transfer between considered pairs of differential elements.

$$
F_{1-2}=\frac{1}{A_{1}} \int_{A_{1} A_{2}} \frac{\cos \theta_{1} \cos \theta_{2}}{\pi S_{1-2}^{2}} d A_{2} A_{1}
$$

It was necessary to take additional assumptions for the simulation:

- $\quad$ the size of microbolometer matrix was $9,6 \times 7,2 \mathrm{~mm}^{2}$, due to the resolution of $284 \times 288$ and pixel pitch $25 \mu \mathrm{m}$,

- $\quad$ the number of $\mathrm{dA}_{1}$ differential elements in $X$ axis is 384 , equal to the number of columns in the matrix,

- the number of $\mathrm{dA}_{1}$ differential elements in $Y$ axis is 288 , equal to the number of rows in the matrix, inner and outer radius values of the ring emitter were the variables in simulations,

- spotlight emitter radius was $1 \mathrm{~mm}$,

- $\quad$ active area of emitters were parallel to the matrix surface, and localised as shown in Figure 3.
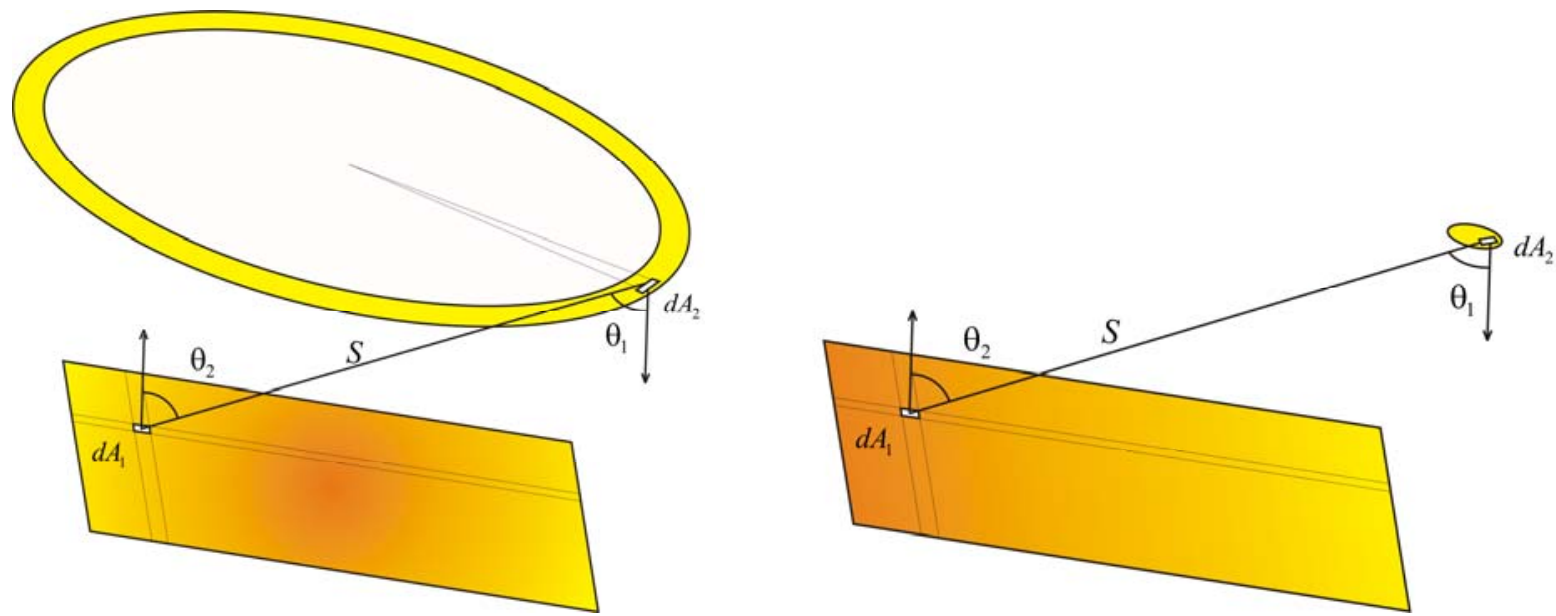

Fig. 3. The model of the a) ring, b) spot emitter and the matrix

First stage of the simulations was to determine the optimal distance values between the emitter and the matrix. Keeping this distance should provide the highest possible illumination evenness as well as possibly high energy. During the simulations, individually for each emitter it was necessary to determine the overall configuration factor value for every $\mathrm{dA}_{1}$ element localised in central (case 1) and border (case 2) row of the matrix. This procedure was repeated for distances from 0.1 to $25 \mathrm{~mm}$ (or $50 \mathrm{~mm}$, depending on the emitter type).

As a result of simulations for ring emitter of exemplary inner radius of $8 \mathrm{~mm}$ and outer $9 \mathrm{~mm}$, there are maps of illumination intensity of center (case 1) and border (case 2) rows of the matrix shown in Figure 4 a and b respectively, versus the distance between the emitter and the matrix. In the first case it may be observed that the lowest changes appear in the distance of about $12 \mathrm{~mm}$, while in the second case - about $10 \mathrm{~mm}$. It means that this exemplary ring emitter of inner radius $8 \mathrm{~mm}$ and outer $9 \mathrm{~mm}$ should be placed 10 to $12 \mathrm{~mm}$ from the matrix to illuminate it as evenly as possible.

Due to the subjective character of above results, the authors conducted the second stage of simulations, which enabled qualitative assessment of required distance values. It based on the determination of two parameters: mean value of configuration factor in the row, and the factor of row illumination unevenness (understood as maximum value of configuration factor divided by the minimal value, and lowered by value of 1 ). The results of quantitative analysis are shown in figures 5 to 7 , and they are coherent with qualitative observations. Dotted lines are for distances beyond the 
boundary condition, which means that the emitter couldn't be localized there as it would act as an obstacle in the way of radiation from the lens, or simply the radiation from the emitter wouldn't be able to reach the matrix.
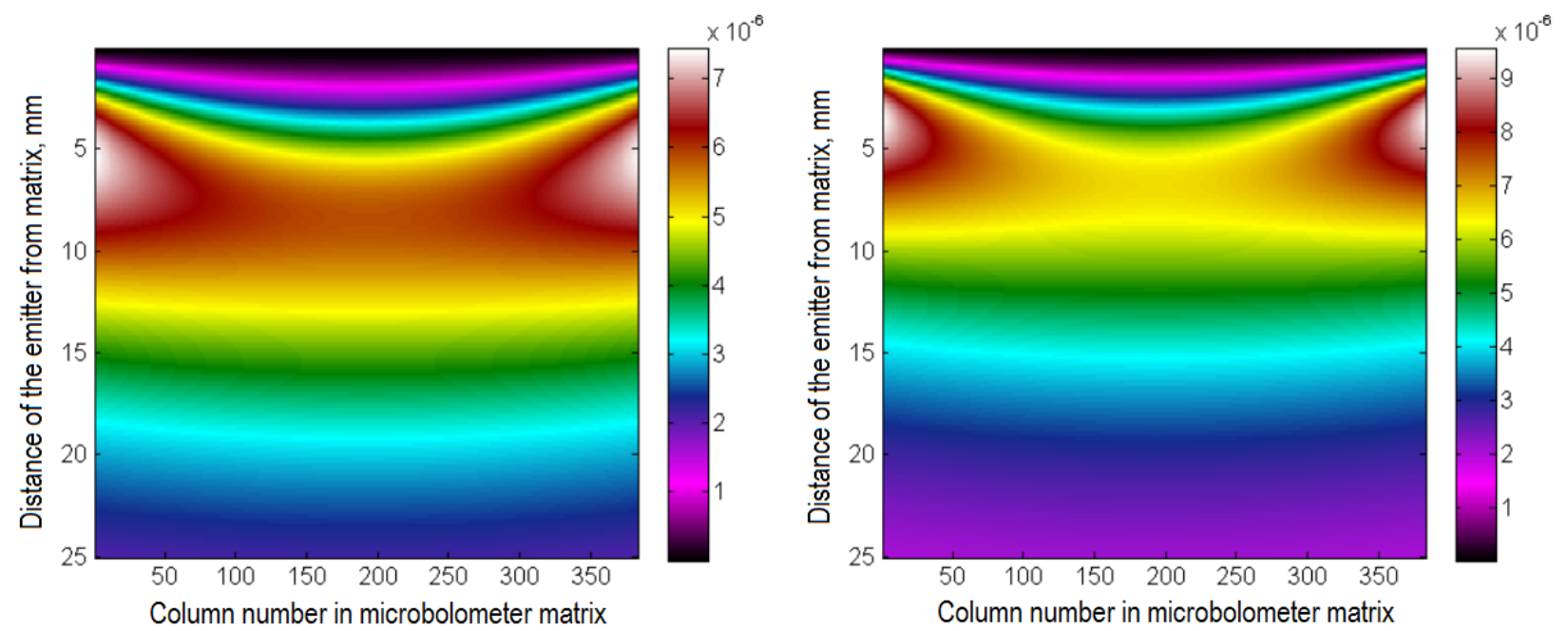

Fig. 4. Simulated illumination unevenness and mean energy versus the distance between the ring emitter and the matrix

Considering figure 5 it may be observed that the best illumination evenness takes place for ring emitter of inner radius $16 \mathrm{~mm}$ localized about $23 \mathrm{~mm}$ from the matrix. But the illumination energy is not quite high then. To increase this energy it is possible to increase the outer radius to $23 \mathrm{~mm}$, and it also improves the illumination evenness - figure 6 . The distance has to be in this case equal to about $26.4 \mathrm{~mm}$. In case of spot emitter it was impossible to obtain even matrix illumination - figure 7 . Next, using values determined during this research (table 1), the authors simulated the illumination of the whole matrix - figure 8 .

Table 1. Optimal values found during the simulations

\begin{tabular}{lcc}
\hline & \multicolumn{2}{c}{ Emitter } \\
\cline { 2 - 3 } Parameter & Ring & Spot \\
\hline Inner radius & $16 \mathrm{~mm}$ & -- \\
\hline Outer radius & $23 \mathrm{~mm}$ & -- \\
\hline Translation & -- & $10 \mathrm{~mm}$ \\
\hline Distance from matrix & $26.4 \mathrm{~mm}$ & $12.5 \mathrm{~mm}$ \\
\hline
\end{tabular}

Authors found out that the matrix may illuminated by the ring emitter very evenly, under the assumption that conditions from the table 1 are in force. It may be even calculated that residual unevenness is about $20 \mathrm{mK}$ (mainly in corners), which is not higher than NETD of a typical microbolometer camera. Therefore in this case in theory it is not necessary to perform the correction of illumination unevenness. But in practice it is advised to compensate for this unevenness, as it is very difficult to localise the emitter with such precision.

It is possible to use smaller ring emitter if it is necessary, then it may be localised closer to the matrix. This is the case in cameras with the lens mounted close to the matrix (low value of focal length). Simulation were carried for different ring emitter sizes, hence it is possible to select the proper size for particular application, and then read the required distance from the matrix in figure 5. 

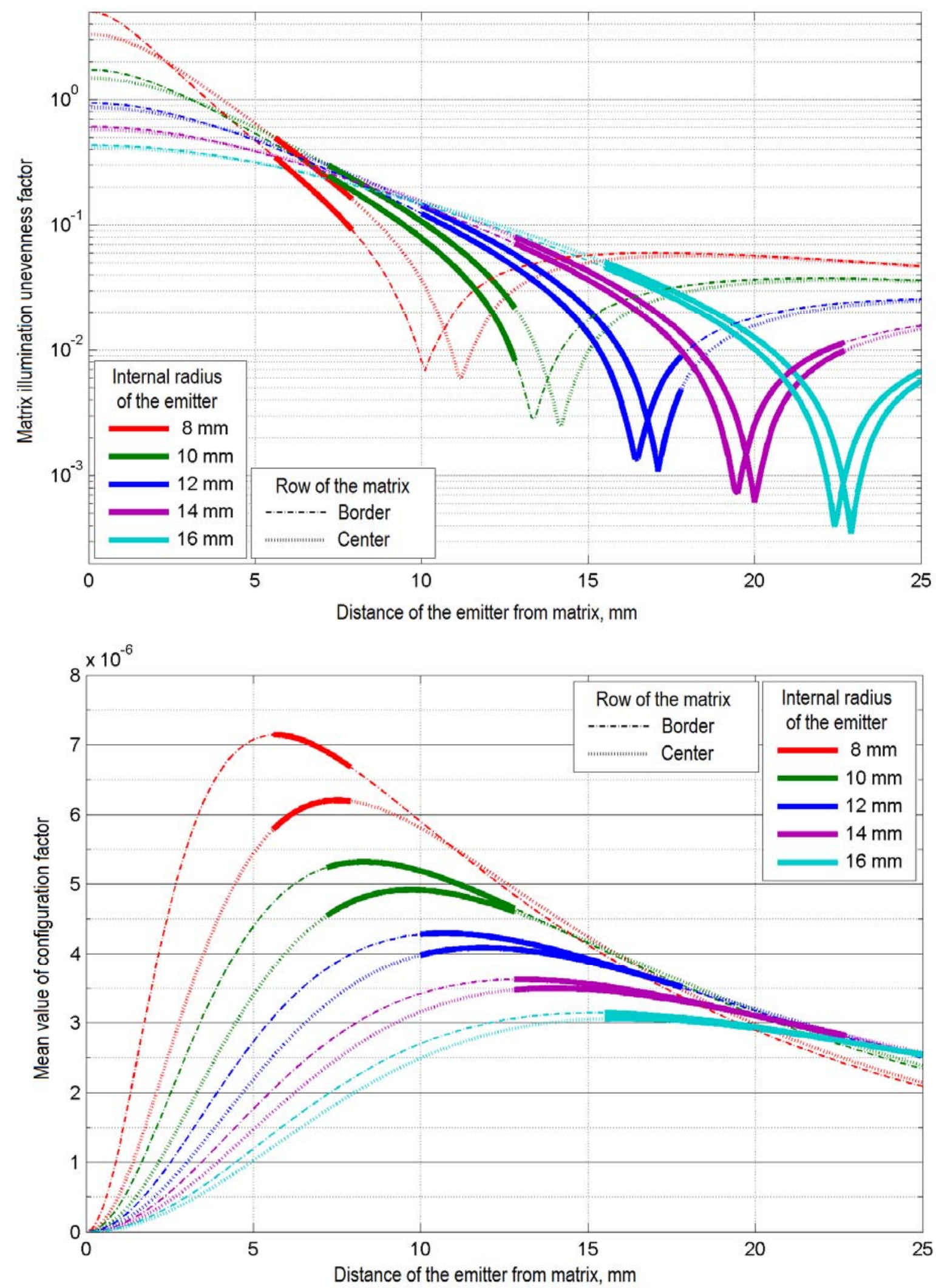

Fig. 5. Simulated illumination unevenness and mean energy versus the distance between the ring emitter (having given values of inner radius) and the matrix 

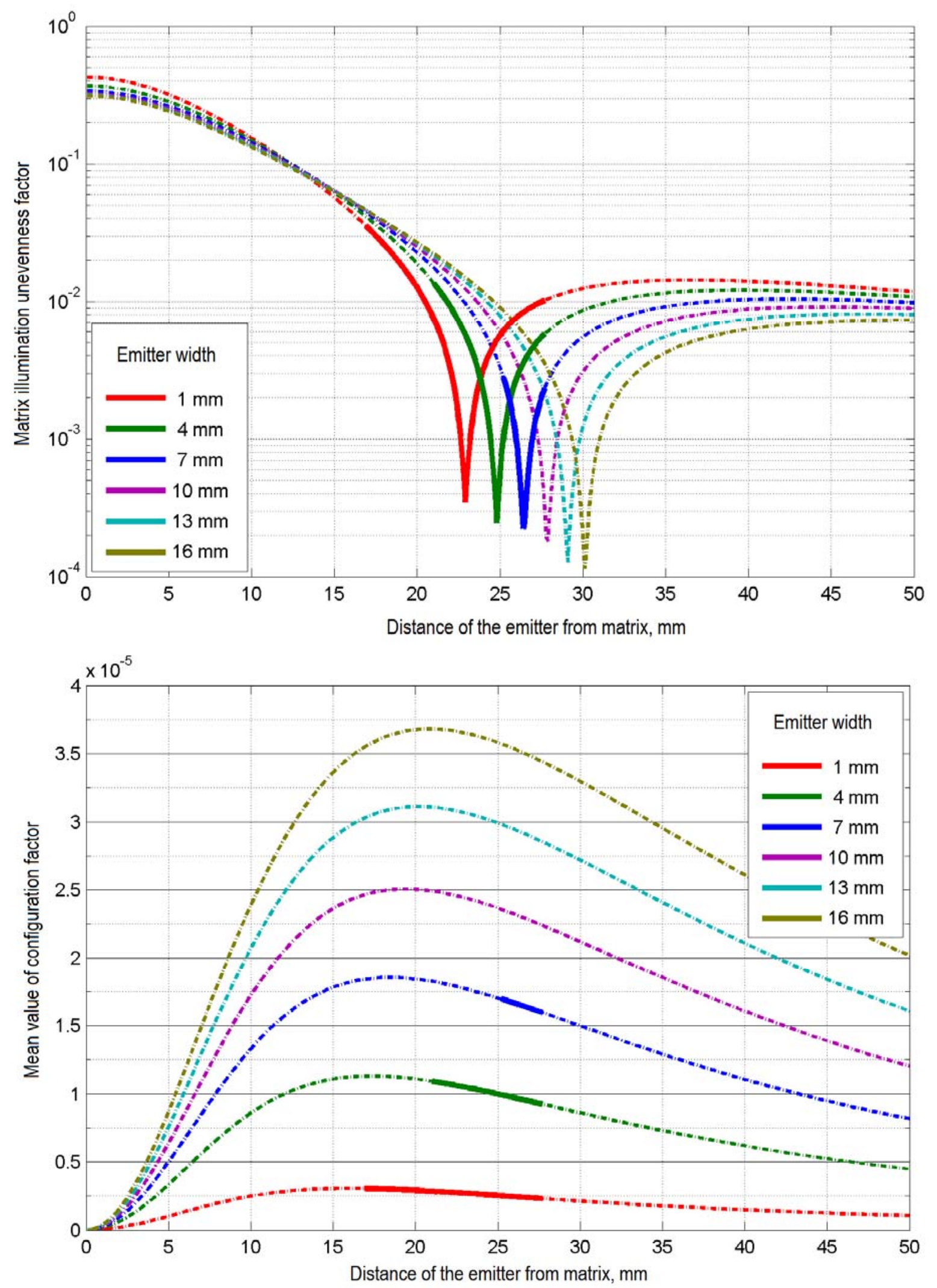

Fig. 6. Simulated illumination unevenness and mean energy versus the distance between the ring emitter (having inner radius $16 \mathrm{~mm}$ and given values of the width) and the matrix 

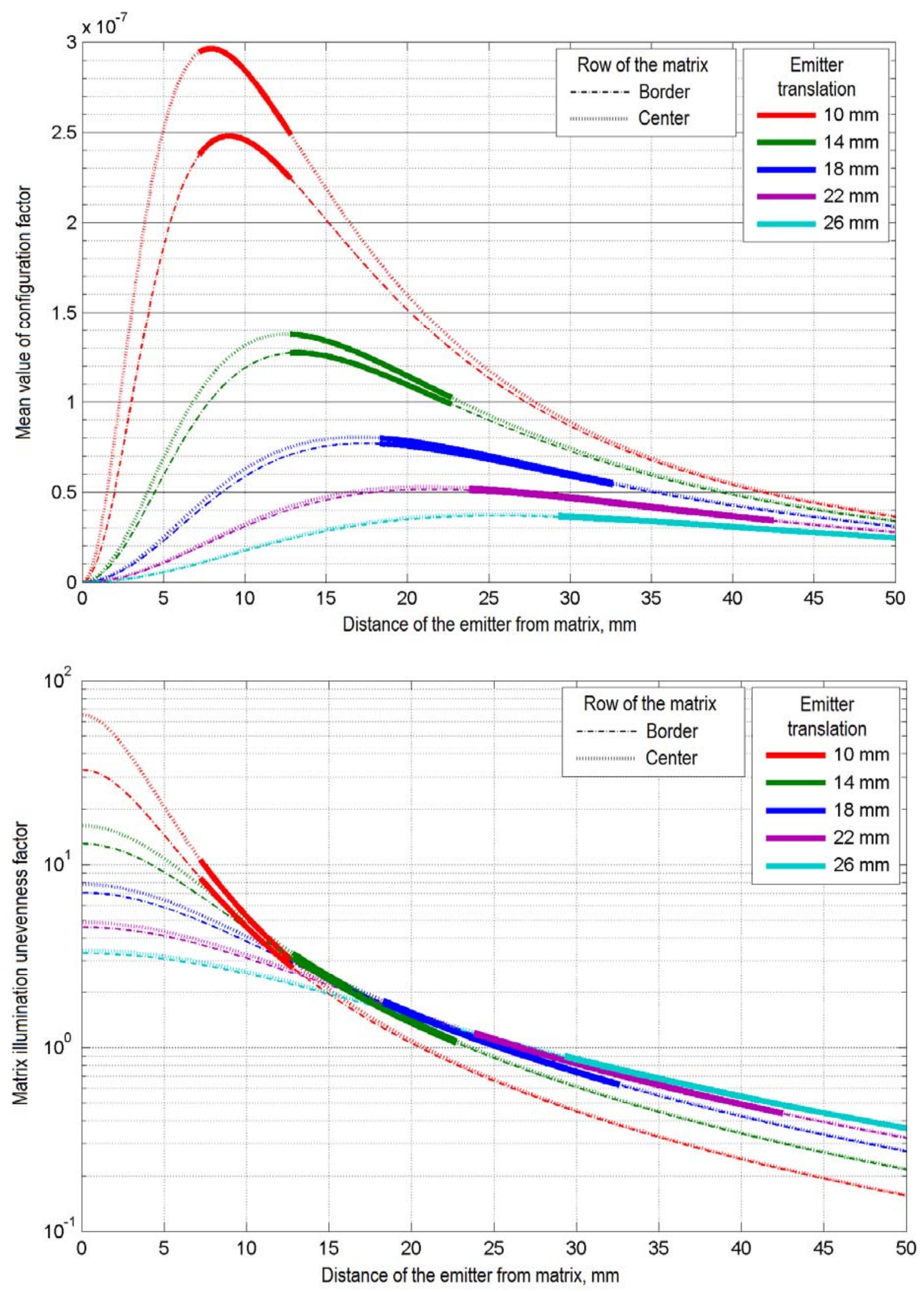

Fig. 7. Simulated illumination unevenness and mean energy versus the translation and distance between the spot emitter and the matrix 

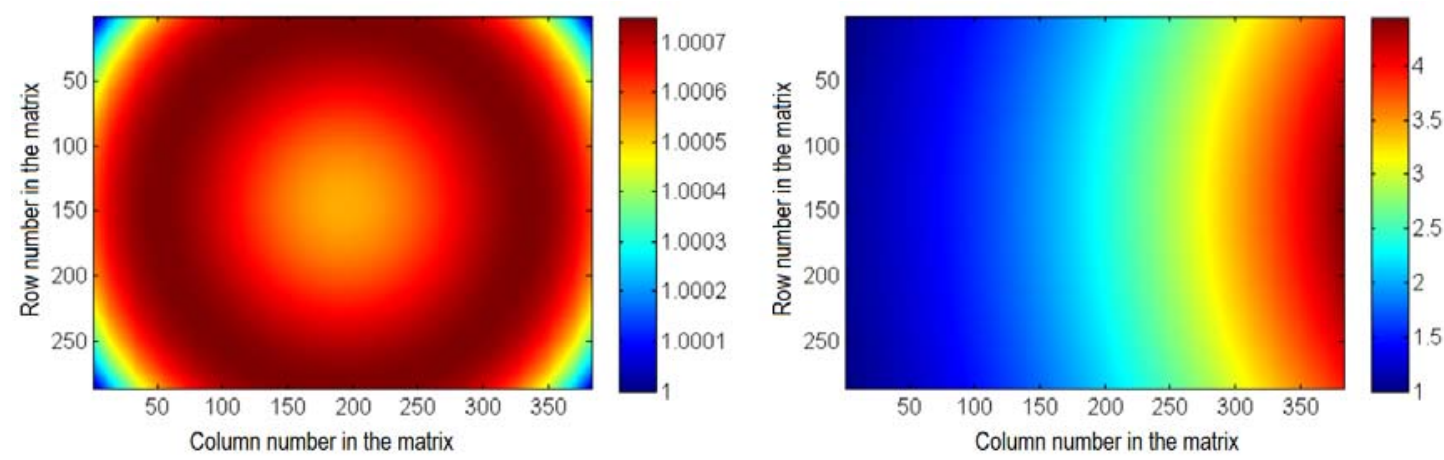

Fig. 8. Simulated illumination pattern for a) ring, b) spot emitter with parameters from table 1

\section{Obtained results}

Results of simulations were used to localize emitters inside the camera. Using these emitters the authors determined matrices of microbolometers' gain values - figure 9. Qualitative and quantitative analysis revealed that in case of spot infrared emitter there were unwanted artifacts (errors) in some of the determined gain values. This problem did not appear in case of reference gain matrix, and the one obtained with ring emitter. This is clearly visible in figure 9c versus figure 9a,b. The conclusion is that the active surface of infrared emitter should be as large, as possible [2].

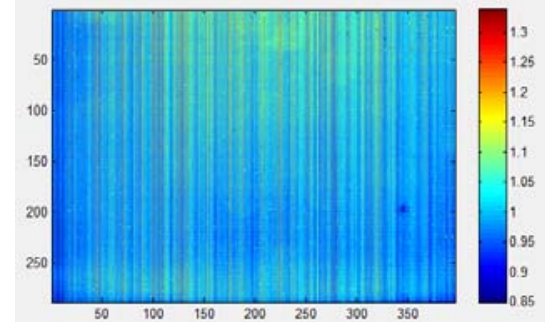

(a)

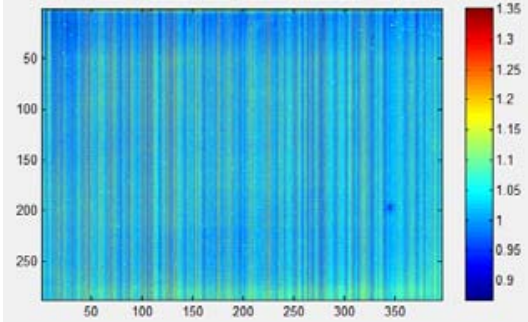

(b)

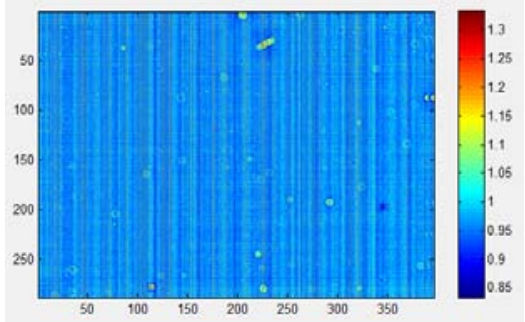

(c)

Fig. 9. Measured gain non-uniformity of microbolometer matrix, obtained with:

(a) two blackbodies (reference matrix), (b) ring emitter, (c) spot emitter

\section{Correction of gain non-uniformity}

For the purpose of gain non-uniformity correction, it is required to periodically (e.g. once per month) use the ring infrared emitter with exactly the same driving voltage. Determined matrices of gain values should be stored in camera memory and compared with the previous ones. If any difference is found, it means that it appeared between the previous and current gain non-uniformity measurement. Hence for the microbolometers that exhibit this difference, correction factors may be determined basing on this measured difference.

\section{Conclusion}

The proposed method for gain non-uniformity determination with the aid of infrared emitter is efficient with ringshaped emitter, provided that illumination unevenness is compensated. Periodical verification of gain non-uniformity in thermal camera enables detection and correction of unwanted gain changes.

\section{REFERENCES}

[1] Więcek B., Olbrycht R., Kastek M., Orżanowski T., Sosnowski T., "The method for gain non-uniformity correction of microbolometer detectors" (in Polish: "Sposób korekcji niejednorodności wzmocnienia matryc mikrobolometrycznych"), patent application no P-387173 dated 02.02.2009 (in Polish).

[2] Olbrycht R., Więcek B., "Influence of infrared emiter on efficiency of gain correction in microbolometer detectors" (in Polsh: "Wpływ rodzaju promiennika podczerwieni na skuteczność korekcji wzmocnienia matryc mikrobolometrycznych"), Measurement Automation and Monitoring, pp. 1127-1129, vol. 57, 10/2011.

[3] International Technology Exchange (Intex), Inc. Pulsed Broadband Infrared Light Source MIRL17-900. http://www.eot.it/pdf/Intex_MILR17_900.pdf 